\title{
High-Speed Differential Track-Hold Digitizer
}

\section{MIISTER}

A. A. Van Hook

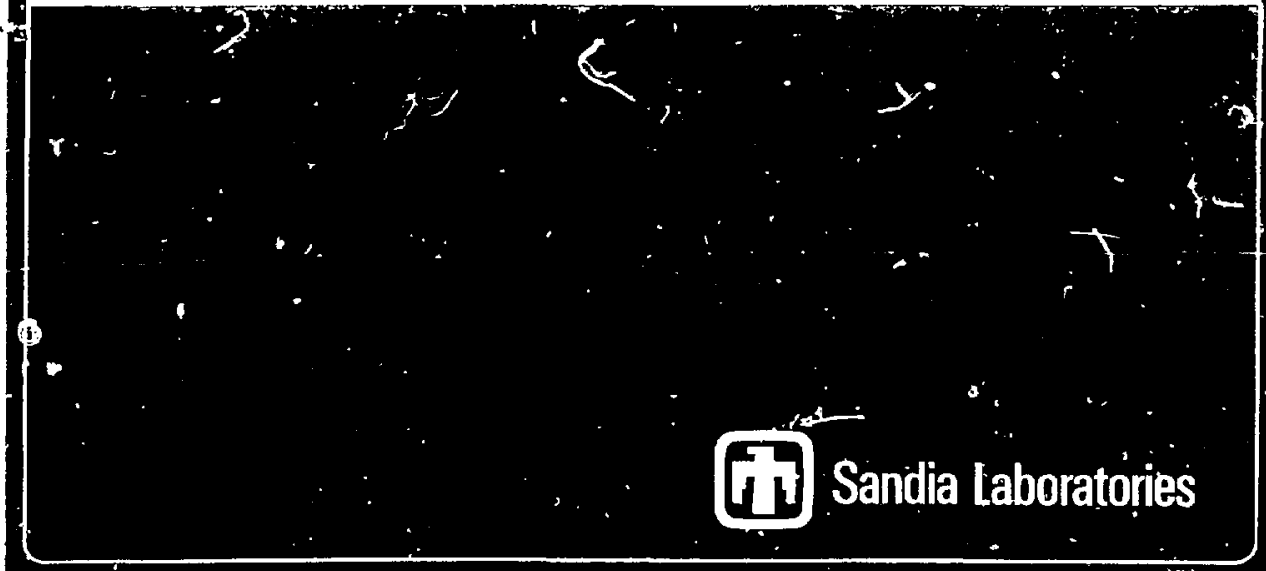


SAND 79-8265

Unlimited Releaco

Printed Decembe: 1979

\author{
HIGH-SPEED DIFFERENTIAL * \\ TRACK-HOLD DIGITIZER
}

d. R. Van Hook

App? ied Physics Division

Sandia Laboratories, Livermore

\begin{abstract}
gbtaining accurate measurements in noisy environments or measurements with slowly varying hackground biases can be difficult, and at Limes impossible, when the background variation has a high fynamir range. Obtaining useful data with resolutions of one part in 10, 00 $(0.01 \%)$ in these types of environments can be simplified through the use of a differential track-and-hold system. This paper describes a differential technique to obtain ground reference correction during realtime high-speed data collection.
\end{abstract}

ऋ Work supported by the IJ.S. Department of Energy 


\section{Introduction}

The design goal of this track-hold/conversiori circuitry was to measure integrated surrent pulses with an accuracy of $0.01 \%$. In:!'t signals were derived from an optic detection system used in monitoring pulses superinposed on à Cir laser beam. The detection system ${ }^{(1)}$ uses a photodiode bridge for first-order noise cancellation. The dioge bridge output, resuiting from the $C$ id laser, is a DC current of $20.5 \mathrm{~mA}$, with low-frequency components carresponding primarily to laser power supply variations. The (rast) signals to be measured are optically coupled to the detection system. The resulting signais occur as wions (FWHM) current pulses repeated at lo0mis intervals, superimposed onto the $D C$ current. This total signal is applied to the input of an Ortec 142A amplifier, which integrates the current pulse onto a lpf capacitor and feeds the resulting voltage to the difierential monitoring and recording system to be discussed below.

The RMS value of shot noise is given by ${ }^{(2)}$

$$
I_{S h}=\sqrt{2 q I_{D C}}
$$

where

$$
\begin{aligned}
& \mathrm{q}=\text { electron charge, } 1.59 \times 10^{-19} \mathrm{C} \text {, } \\
& I_{D C}=\text { direct current in } \mathrm{A} \text {, und } \\
& \Delta f=\text { noise bandwidth in } \mathrm{Hz} \text {. }
\end{aligned}
$$

The integrated shot noise can be approximated by

$$
Q=\sqrt{I_{D C} T q}
$$

where $T=$ time (integration period). This $Q$ represents $2 \pi V$ when a $D C$ current of $0.5 \mathrm{~mA}$ is integrated on a lpf capacitor for 50ns. Thus, the differential track-hold and conversion system has to resolve at least $2 \mathrm{mV}$ to be shot noise 1 isitited. 


\section{The Circuit}

The circuitry is housed in a triple-width Nim madule provided with $\pm 6 V, \pm 12 V$, and $\pm 24 V$ from a standard Nim-powered rack. The construction uses all-ground plane, high-frequency techniques, with major inner connections done with coax and SMA connectors.

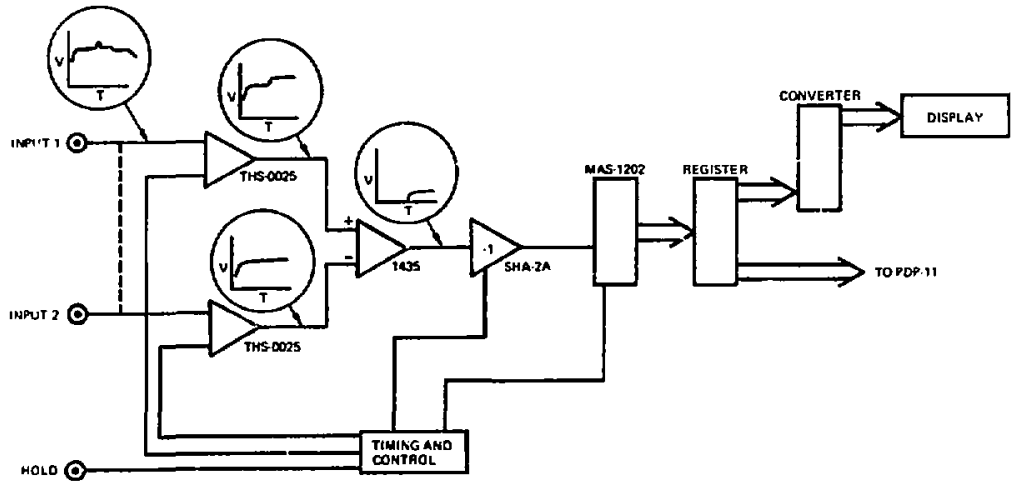

The "front end" (see Figure 1) of the differential system consists of two Computer labs THS-0025 Track-and-Hold Modules, with acquistion times of $15 \mathrm{~ns}$ and linearities of $0.01 \%$. The input range is $\pm 2 \mathrm{~V}$, leading to a resolution Iimitation of $200 \mu \mathrm{V}$. The output of each THS-0025 is connected to a separate input of a Teledvne Philbrick Model 1435 highfrequency, fast-settling operational amplifier. The 1435 has a settiing time of 70 ns to $.01 \%$. The output of the 1435 is fed to a third track-andhold amplifier, an Analog Device Model SHA-2A. The SHA-2A in turn drives 
a Computer Labs analog-to-digital converter, model MAS-1202. This converter generates a 12-bit digital binary word in $2 \mu s$, to avoid errors due to the droop rate of the SHA-2A. The $2 \mu$ s conversion time also sets the repetition rate limitation on the system of $500 \mathrm{KHz}$ (more than adequate for the $10 \mathrm{~Hz}$ data rate.)

The binary output from the MAS-1202 is fed via the rear panel to a PDP-11 for data storage and manipulation. The binary data are also rauted internally to a series of SH74185 TTL memories, where the data are converted to BDC for a front panel 4-digit LEO display.

The other stipport circuitry consists of timing modules for signal control and power supplies used to convert Nim power to module power.

\section{Operation}

The basic design principle of the circuitry is that of differential noise subtraction. The front end modules (with their inputs in parallel) normally are operated in "track" mode, i.e., the outputs track or follow the inputs. Since (normally) the inputs look at the same input signal voltage and the outputs are subtracted, the resulting output of the 1435 is zero.

With the anticipation of a pulsed signal, one of the THS- 0025 madules is conmanded to "hold", thereby storing a reference voltage. After the signal propagates through the other module, it too is "frozen", storing the sum of the reference voltage and the signal pulse. The magnitude of the reference voltage will not be exactly the sane in both amplifiers, since a finite time has elapsed between the two hold commands. This time delay is variable and can be set to some compromise between noise cancellation and signal rise time: i.e., the delay has to be long enough to allow the input signal to get through the second amplifier; otherwise, it will be clipped. 
The 1435 output is the difference of the two input voltages, or simply the pulse stgnal with respect to ground. This ground-referenced pulse can be of either polarity, depending on whtch module is triggered first. In practice, this trigger order is critical, since the A/D converter can respond only to positive pulses. The output of the 1435 must be negative, since it is inverted by the SHA-2A before entering the converter, thus satisfying the polarity requirenents of the HAS 1202. Internal PC switches determine this triggering order and also the time between the two trigger points, allowing for a higher degree of noise cancellation during shorter putses.

The SHA-2A sample/hold was needed to compensate for the high droop rates of the THS-0025 (5mV/us); otherwise, a signal integrity would suffer because the converter could not fojlow the input. The SHA-2A is also used to amplify the differential signal by a factor of 2.4 , to allow the least significant bit of the digftizer to represent ImV. The MAS-1202 has a full-scale input of InV, giving the LSB (least significant bit) a value of $10 / 2^{12}$ or $2.4 \mathrm{mV}$. The 1.53 voltage was set to be conveniently larger than the resolution accuracy limitation of $200 \mathrm{NV}$ of the front end, and the (worst-case) noise fiom the front end.

The system can be used in a single-ended mode by grounding one of the inputs of the front and, or in a biased single-ended mode by applying a $O C$ offset to either input, providing that the polarity to the $A / D$ converter is posftive. The result is a gated fast digitizer with a 0 to $\pm 2 v$ input range.

\section{Limttations}

The major limitations of the existing destgn consist of system noise. temperature drift, and pedestal error. 
The system (or rms) noise to the $A / O$ converter is comprised of the sum of the squares of contributions from the individual modules -- roughly $310 \mathrm{~V}$-- which sets a minimum weight of the least significant bit.

The temperature drift in the individual modules, although specified quite 10w (typically less than $30 \mathrm{ppm} / \mathrm{C}^{\circ}$ ), creates a total system variation of 1 to $2 m V$ during warm-up. This warm-up time can be as much as 30 minutes.

The pedestal or trigger feed-through error can be very troublesome. Several millivolts of trigger pedestal can be injected through the different sample/hold pulses from their respective trigger pins. Once the DC calibration has been accomplished (gain and zeroing) for each of the modules, the pedestal error can be subtracted by using the offset of either the last track-and-hold amplifier or the A/D converter. The visibility of the pedestal does lead to one advantage during initial setup. By examining the various test points during setup, it is possible to determine exactly when each module is triggered, to allow for fine tuning of the system timing. Summary

The differential system provides real-time noise cancellation with a dynamic range of $4 V( \pm 2 V)$ and a resolution of $500 \mu V$ detectable by one-half the LSB. It also provides front panel test points for ease of the initial timing setup and calibration veriffcation. The operator can also observe a digital display on the front panel during real-time data acquisition. The overall data rate is 1 imited to $500 \mathrm{kHz}$ with the proper TTL level triggering.

The system is currently being used on two different research projects:

1. To acquire spontaneous Raman temperature and density measurements in an operational internal combustion engine with equivalence ratios greater than one. (Previously limited to lean mixtures, f.e. equivalence ratios $<0.8$.) 
2. Single pulsed data 7 Imited to shot noise during stimulated Raman and inverse Raman diagnostic development. 
Performance Characteristics

Resolution

Accuracy

Nonlinearity

Conversion Time

Repetition Rate

Common Mode Rejection

Analog Input Characteristics

Input Range

Input Impedance

LSB Weight

Over Vol tage

Incode Command

Logic Levels

Logic ' 1 ' Input Current

Logic ' 0 ' In Current

Differential Separation

$\operatorname{Max}$

Min

Outputs

Parallel Data

Fornat

Logic Levels

Oigital nisplay

Full Scale

Min Display Fesolution
12 bits

$.1 \%$ of FS \pm LSB

\pm LSB

2hS

$500 \mathrm{KHz} \operatorname{Piax}$ (1ess

differential separation time)

$.07 \%$

$\pm 2 \mathrm{~V}$ (0 to $\pm 4 \mathrm{~V}$ with $\mathrm{OC}$ offset)

1 Megohm

$1 \mathrm{mV}$

$\pm 4 \mathrm{~V}$

TTL

50,A (Incode)

$-2 \mu A$ or 0

100 NS

0 NS

12 lines of Binary plus 'data ready' line (held until next encode command)

Staniard TTL up to 10

loads per line

(inverted logic)

4096 (mV)

0001 (mV) 
Acknowledgment

The author wishes to thank $L$. A. Rahn for technical suggestions during this project. 


\section{:REFERENCES}

1. L. A. Rahr and P. L. Mattern, "Coherent Raman Spectroscopy for Combustion .pplications," SPIE, Vol. 158, 1978.

2. C. D. Motchenbacker, F. C. Fitchen, "Low-Noise Electronic Design," J. Wht Tey, Canada, 1973. 


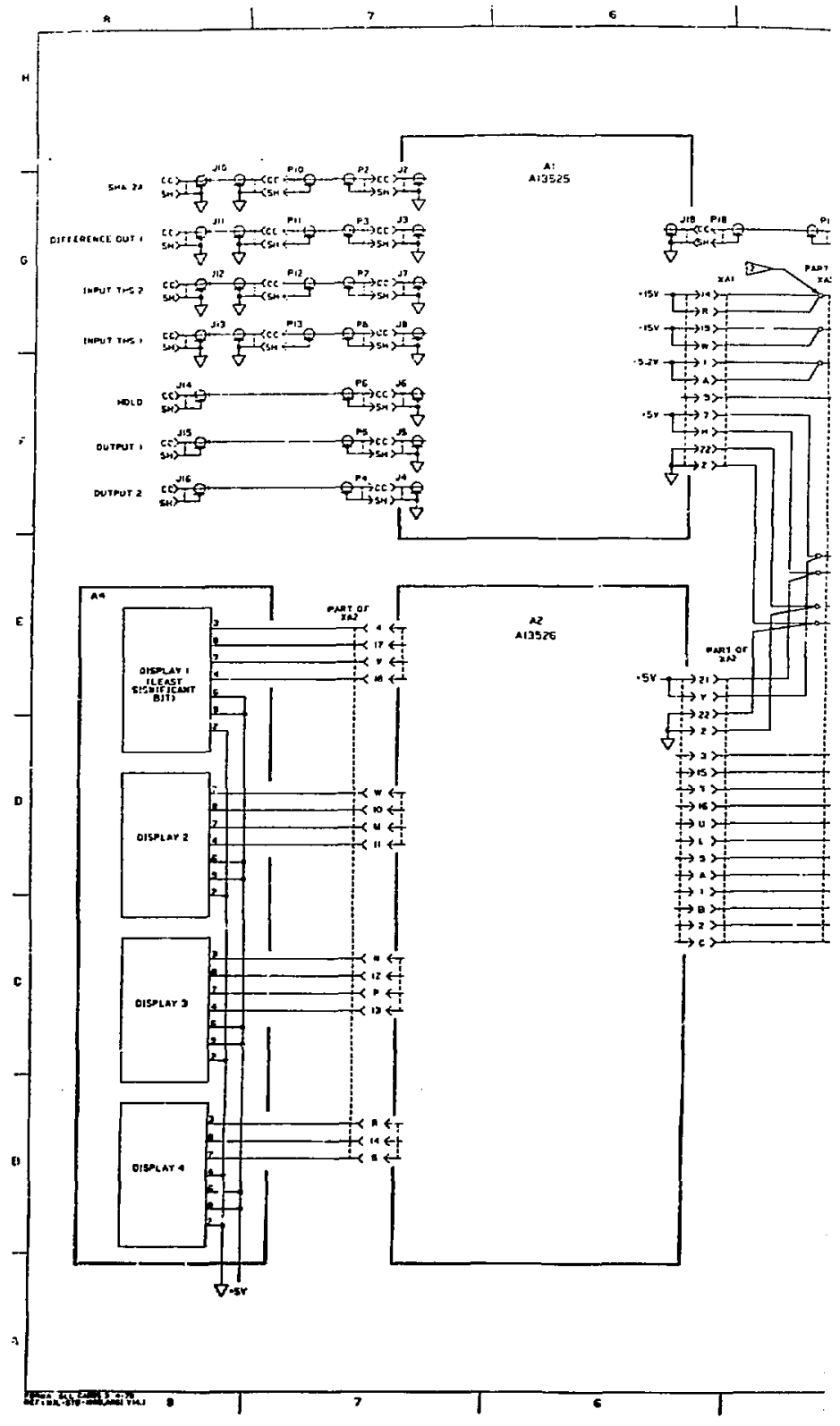


4 3

1 mecers romor

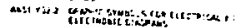

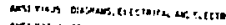

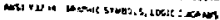

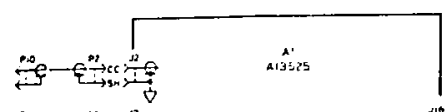

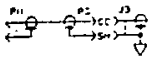
$\stackrel{+2}{T}$ $\stackrel{-2-20}{\longrightarrow}$
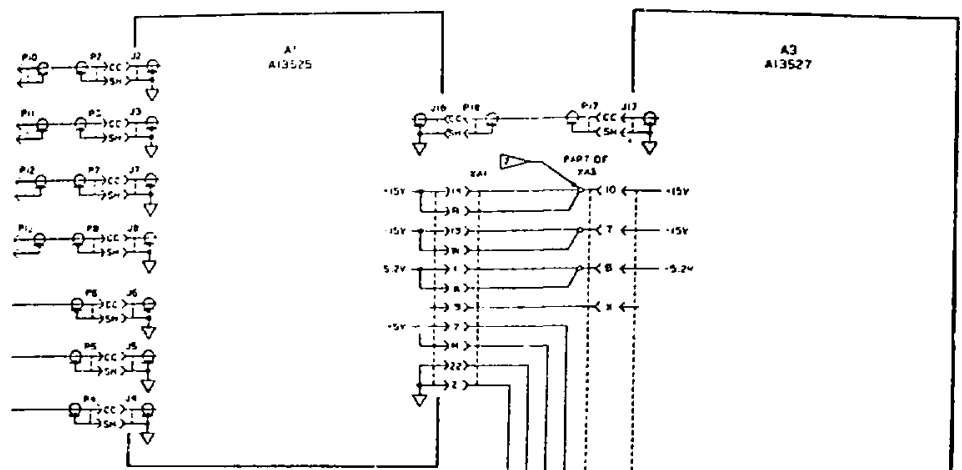

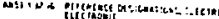

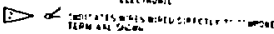
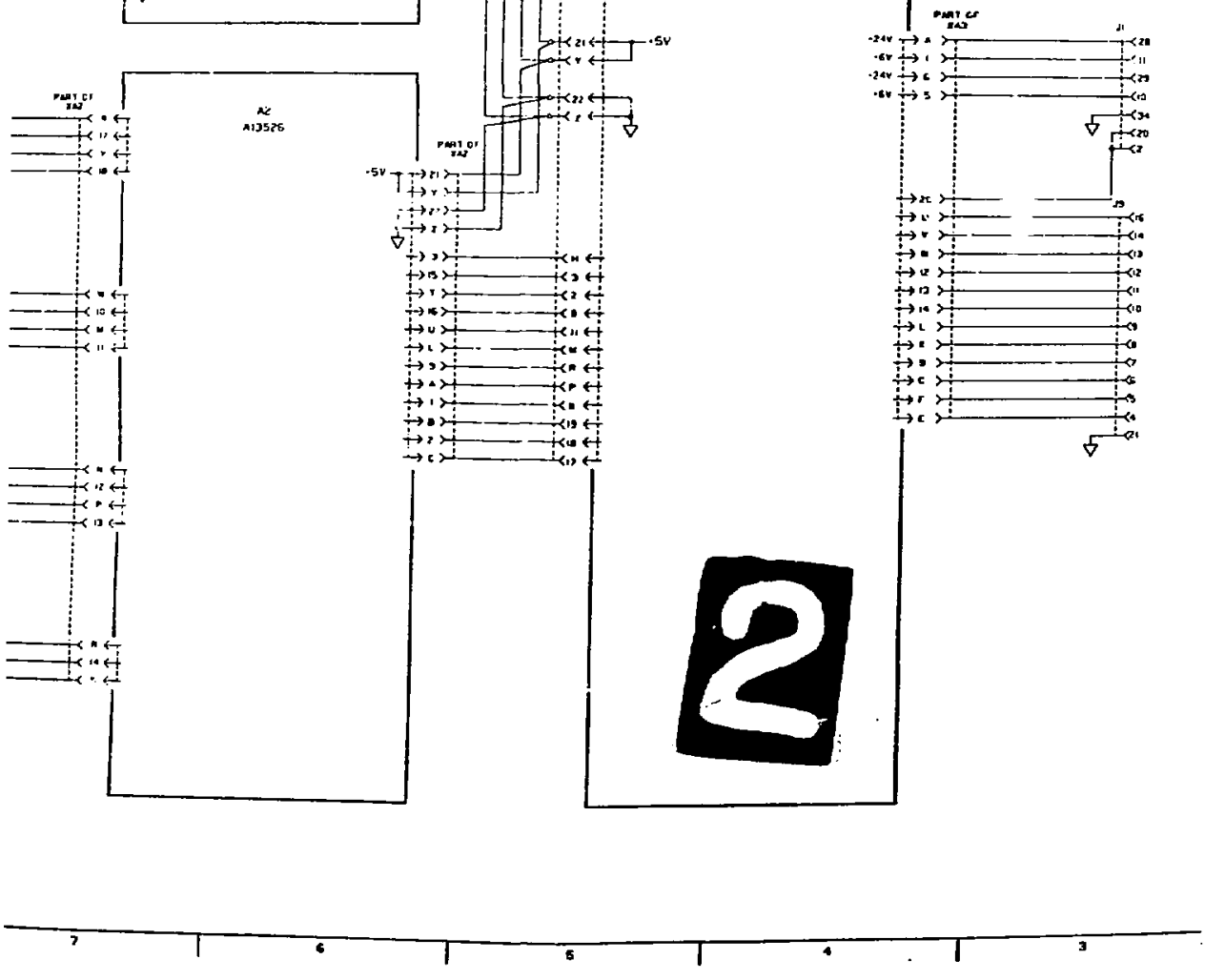


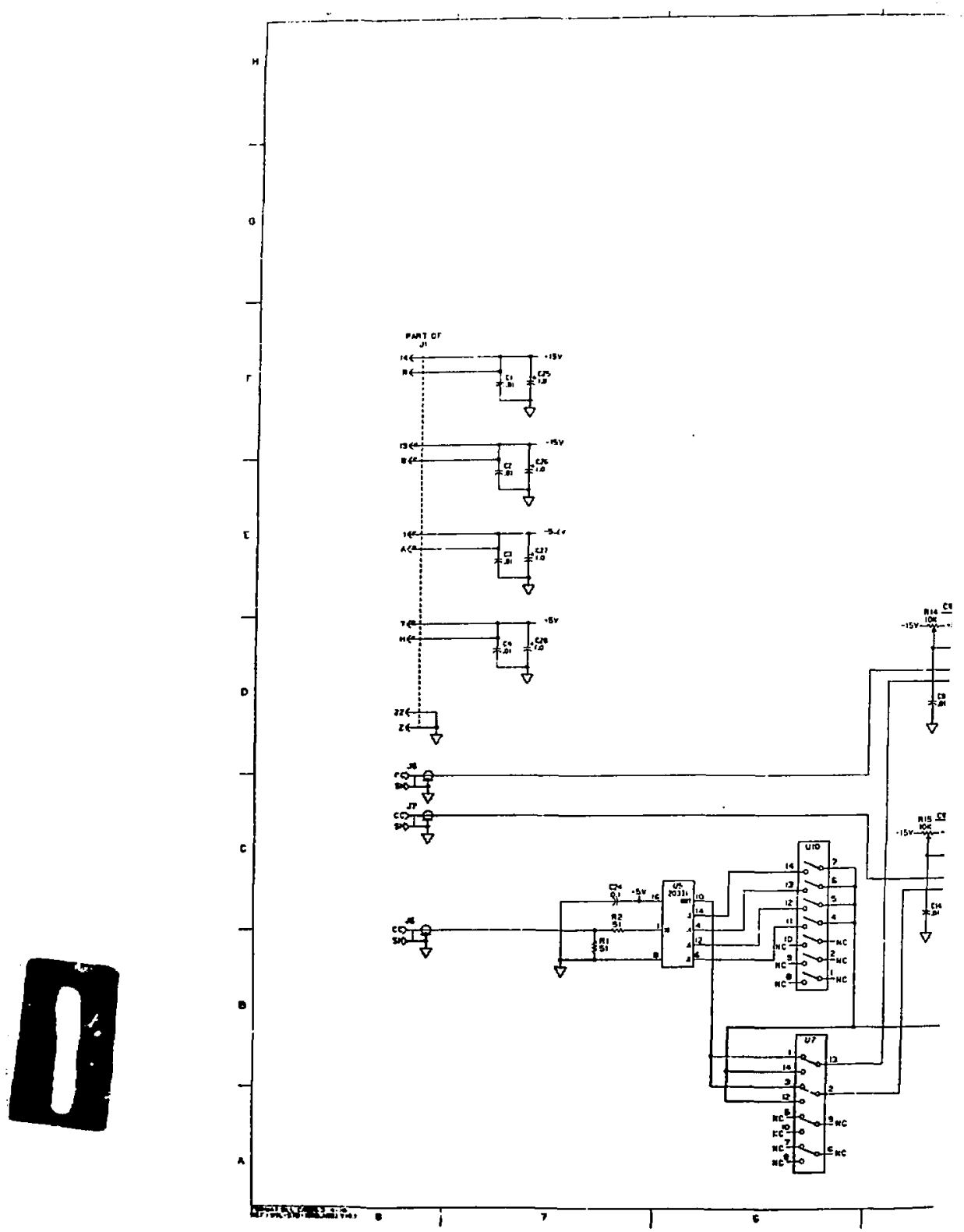




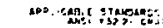

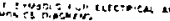

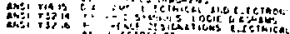

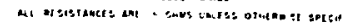

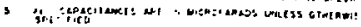

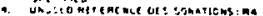

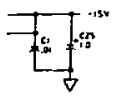

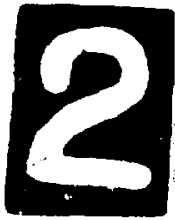

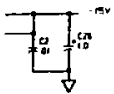
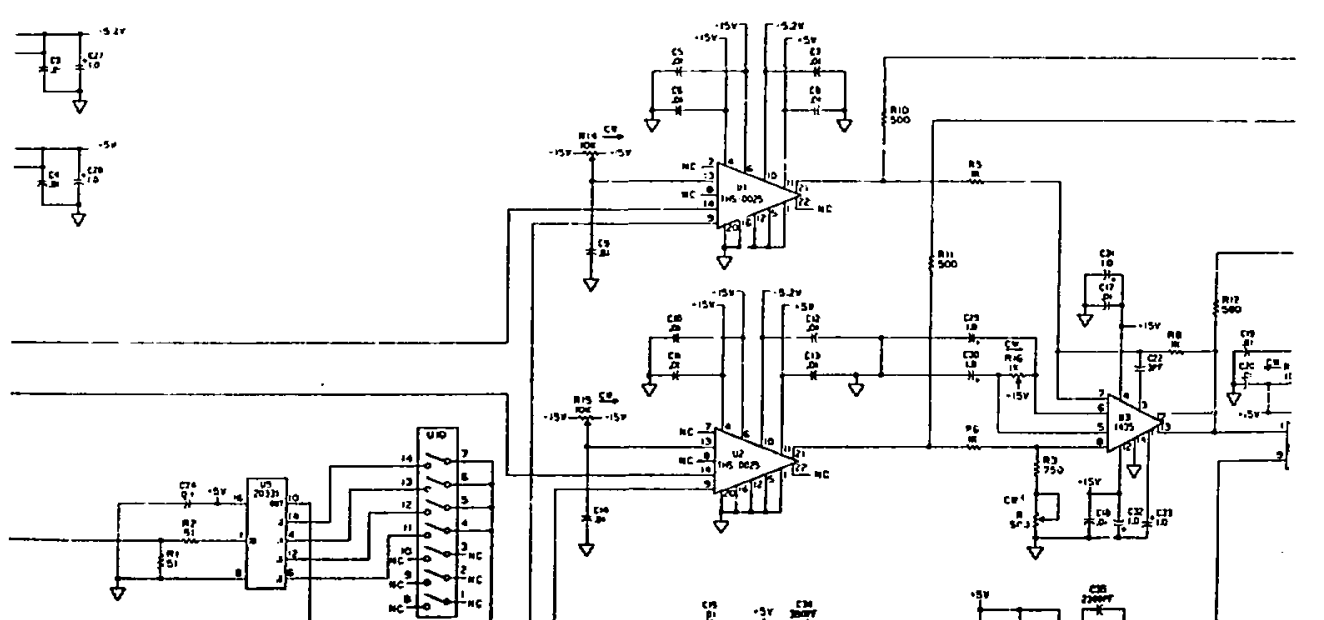


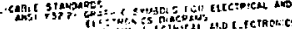

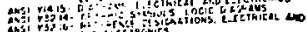

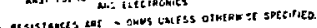

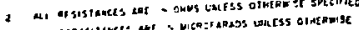

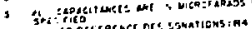
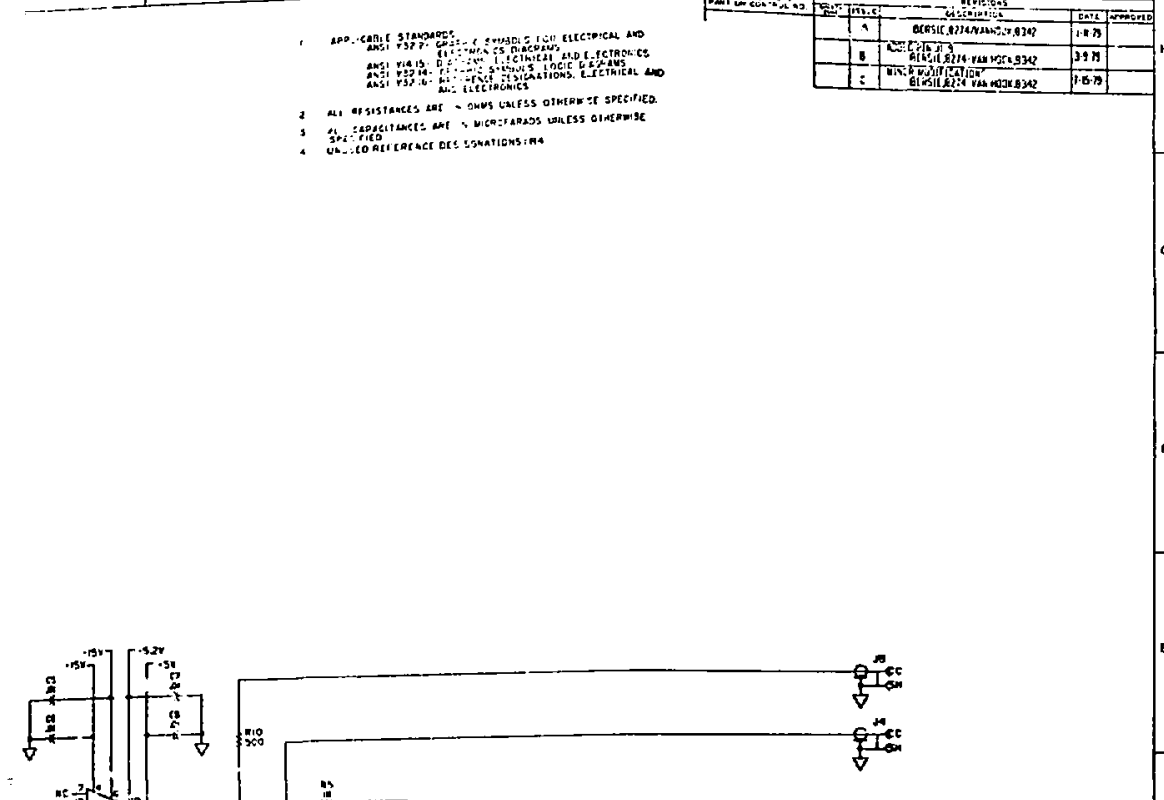

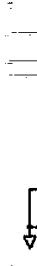

me

,
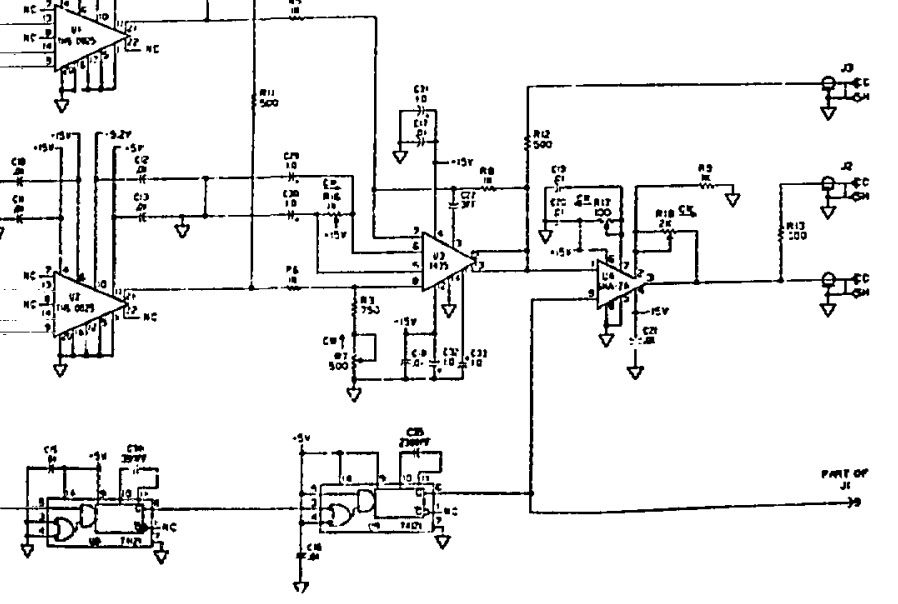

:
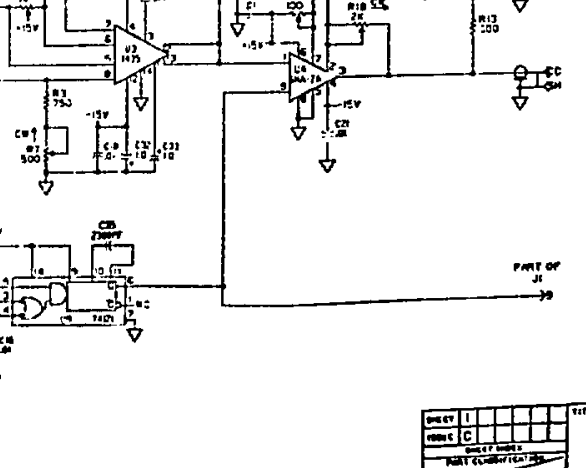

SCHENATIC. TRACK HOLO CIRCUIT 


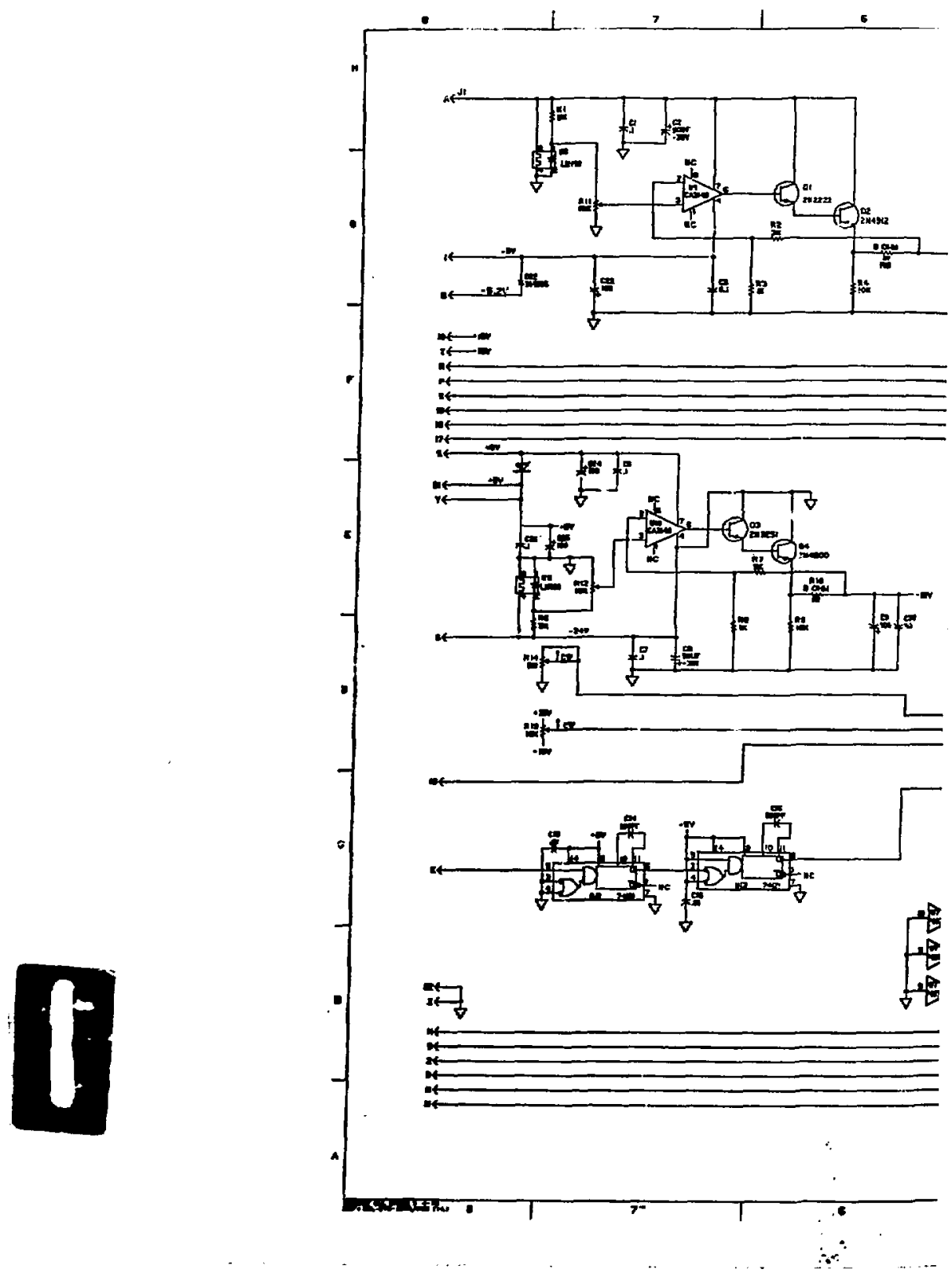



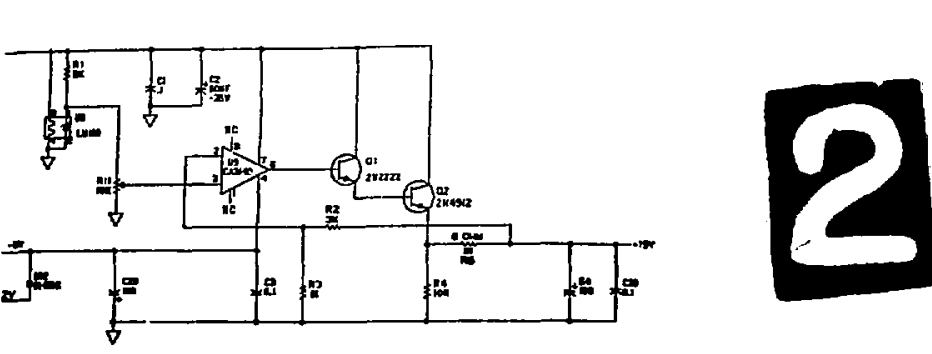

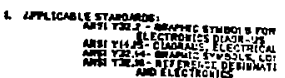

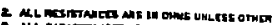

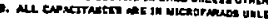

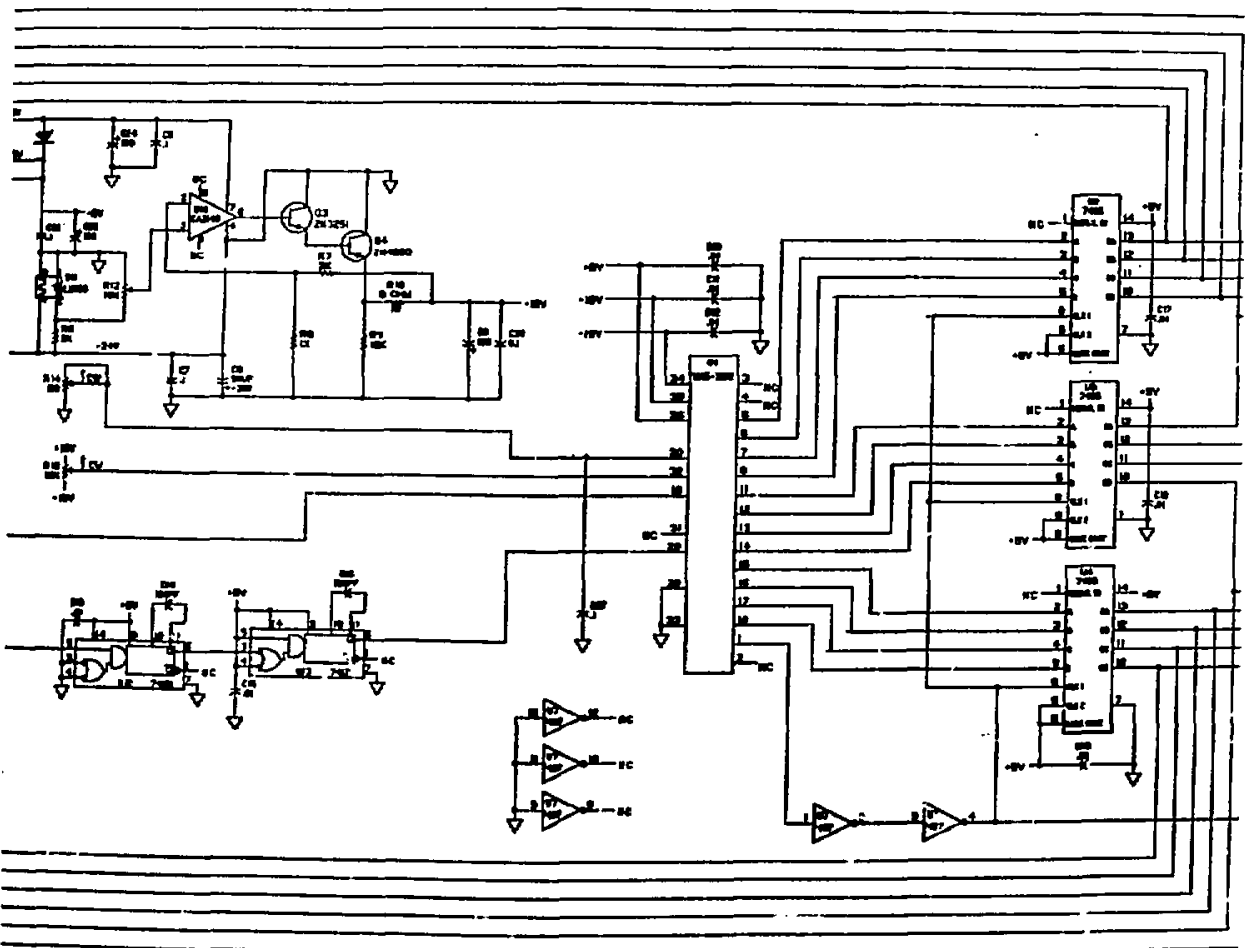

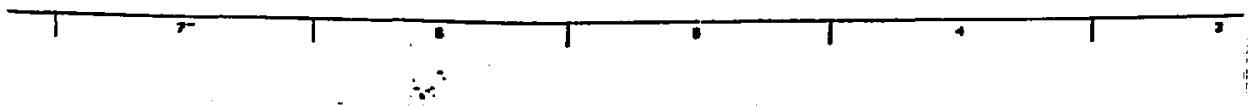



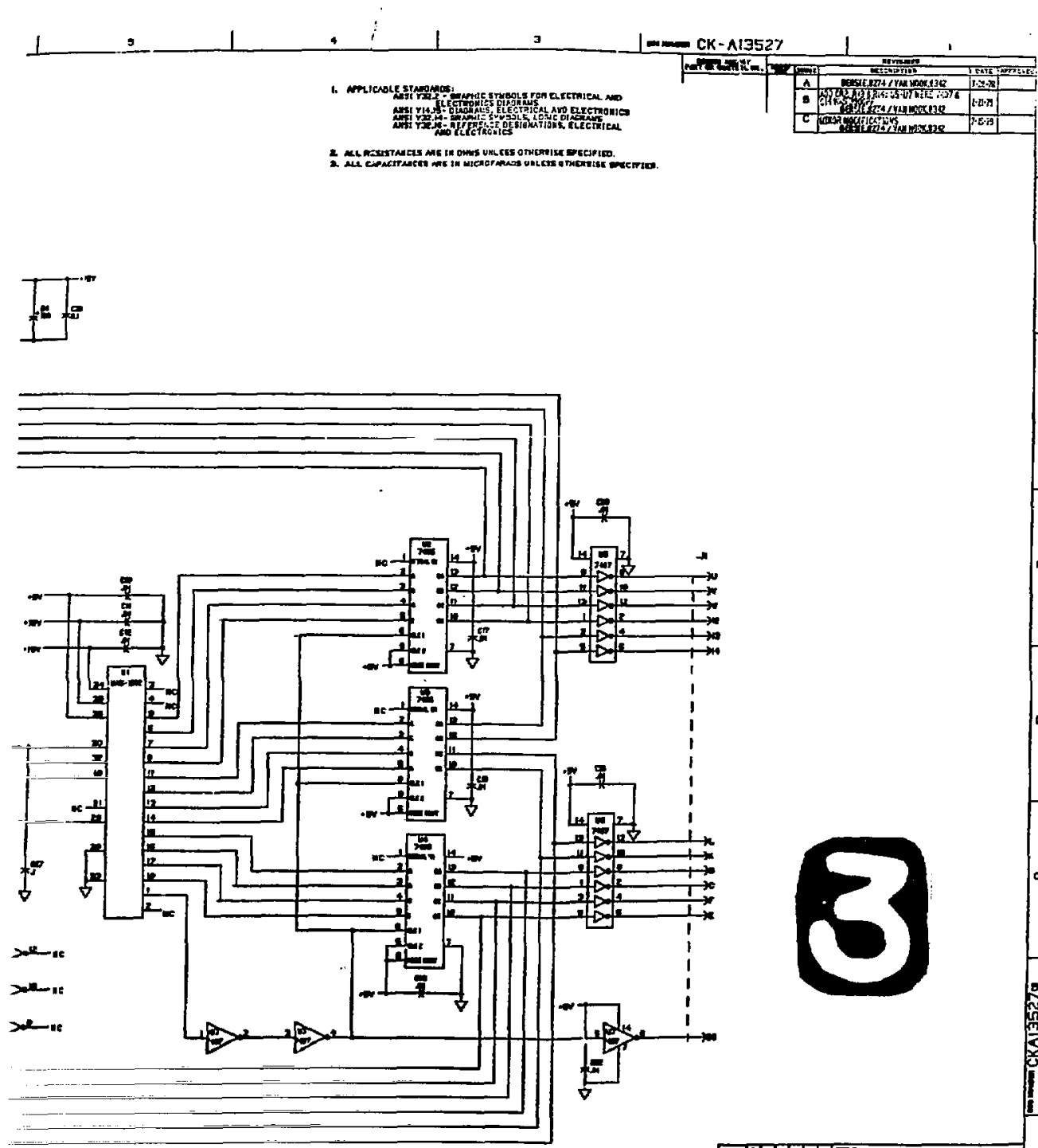


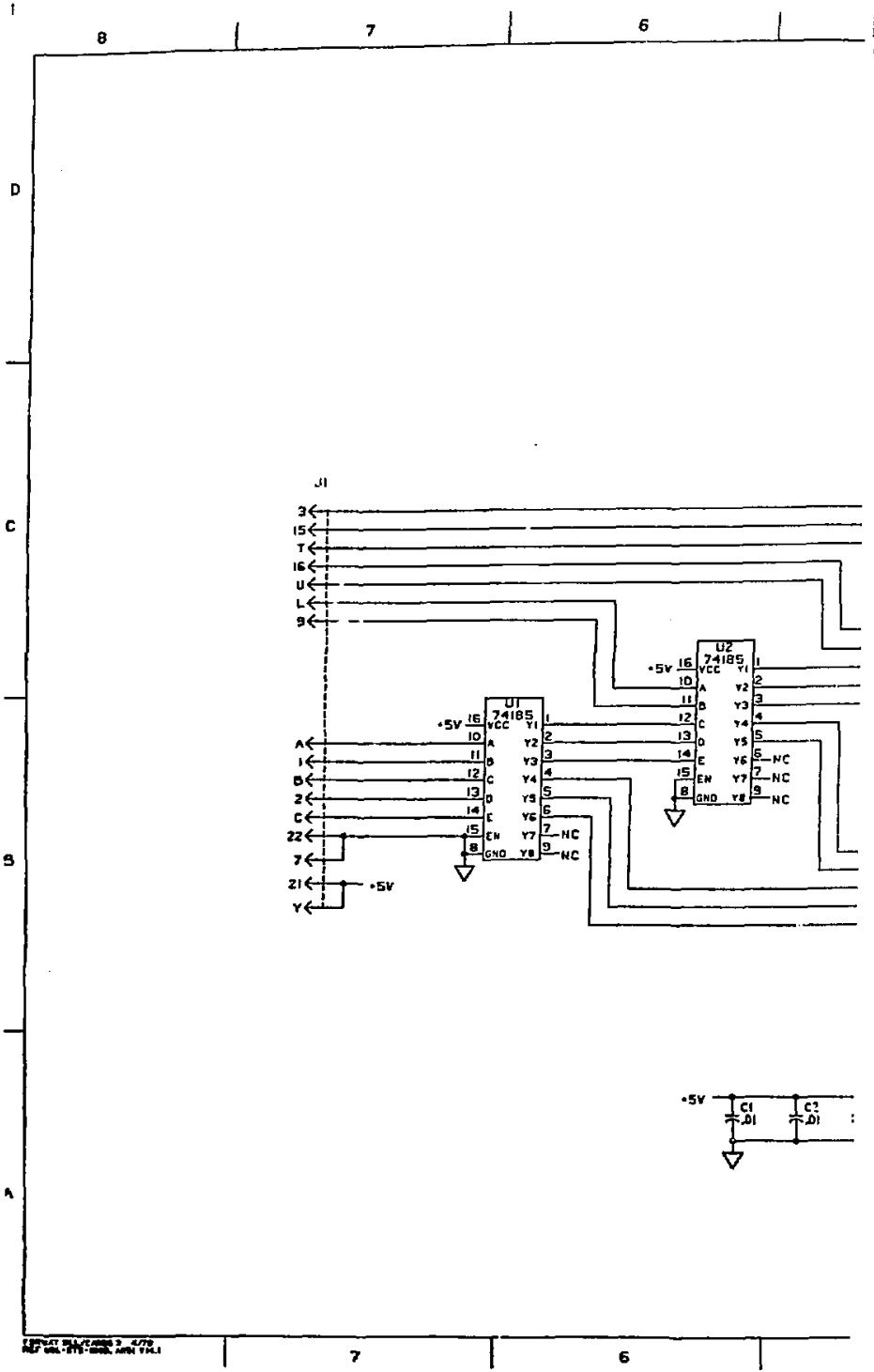




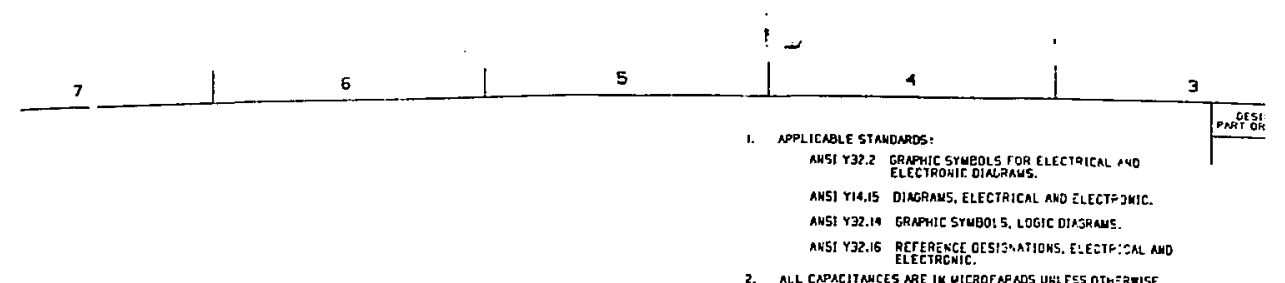

2. ALL CNPACITAHEES ARE IM UICROFAF ADS ULAESS OTMEREISE
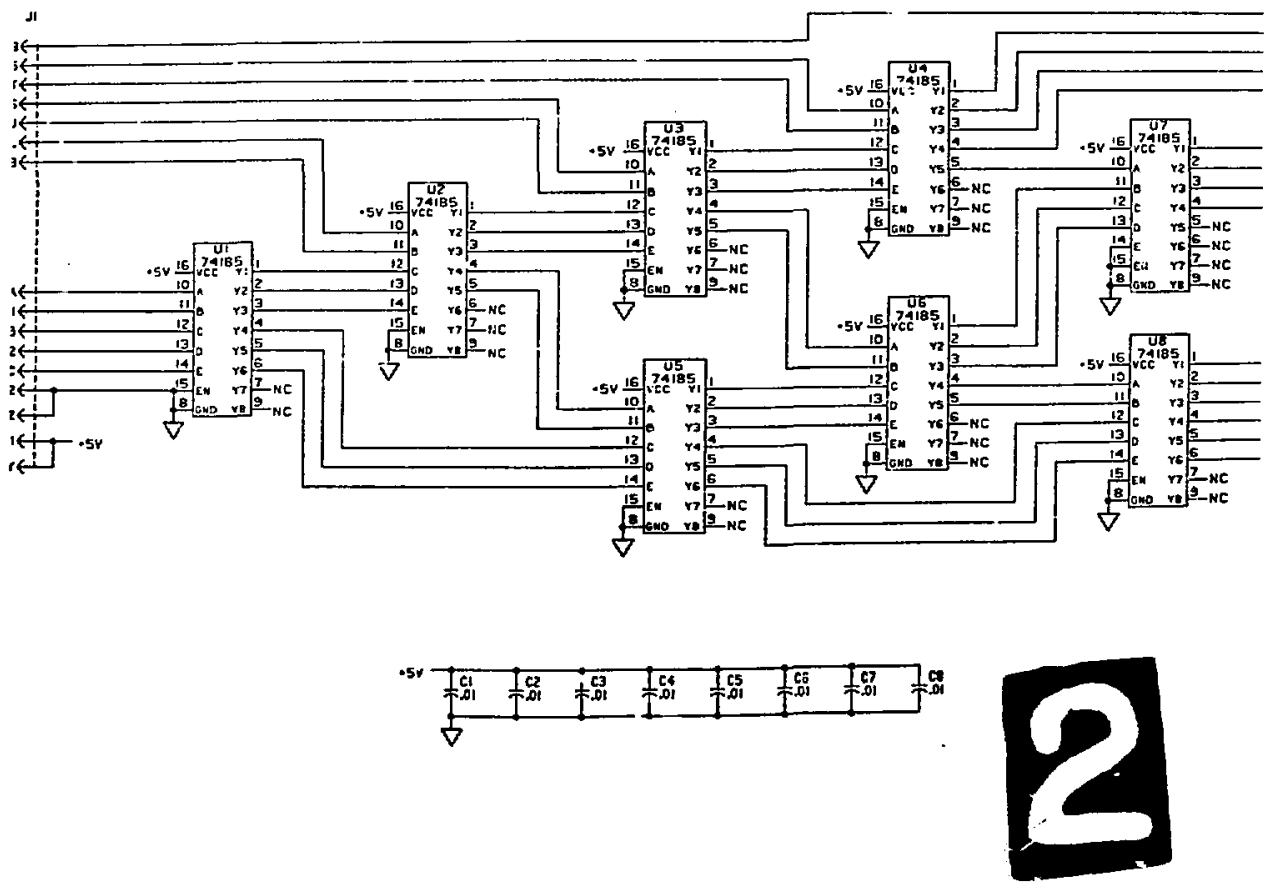
